\title{
Work-Physiological Approaches in Physical Ergonomics
}

According to the International Ergonomics Association (IEA), Ergonomics integrates knowledge derived from the human sciences to match jobs, systems, products, and environments to the physical and mental abilities and limitations of people. Within that broad scope of applying theoretical principles, data, and methods to (e.g., work-place, tool and product) design in order to optimize human well-being and overall system performance, the domains of specialization have changed in the past. Compared to Organizational and Cognitive Ergonomics, the number of research papers dealing with Physical Ergonomics, which were dominant until the end of the last century, has declined in Ergonomic Journals and Conferences, despite the fact that a lot of problems and deficits in work design still exists both in industry and in the service sector.

For the $5^{\text {th }}$ International Conference on Applied Human Factors and Ergonomics (AHFE) fortunately, several papers for a technical session entitled "Work-Physiological Approaches in Physical Ergonomics" could be gathered. Not just simple paper and pencil tests but anthropometric and biomechanical measurements and considerations as well as multi-channel electromyography combined with subjective assessments of experienced subjects (objective data and subject-related approaches) enabled a reliable insight into the essential ergonomic criteria of working tools, work places and products.

For this Special Issue, five extended and revised contributions have been selected.

The first paper by Penzkofer et al. deals with screwdrivers, probably the most wide-spread handheld tools in industry. Measurements of the maximum exertable torque in a pinch and a power grip for pronation and supination were carried out. In addition, subjective assessments of design aspects such as shape, dimensions, material and surface of the handles were recorded. Via multi-channel electromyography, also the physiological costs could be quantified which the muscles of the hand-arm-shoulder system have to pay for working with more or less ergonomically designed handles. These comprehensive data showed a great impact on the user-friendliness of the tools and exhibited only screwdrivers, which had been designed according to ergonomic criteria, as tools that enable a high operational performance (torque strength) at reduced physiological costs, and will not lead to physical complaints in the fingers and the palm of the users' hand.

The paper by Groos and Kluth addresses a special problem in indoor fire-fighting. In order to ensure safe and precise fire-fighting, and that the nozzles work most efficiently, the operating pressure for the water supply may not be chosen just by current administrative regulations and standards which are inconsistent and mostly not based on the results of intensive research. Physiological responses to handling nozzles in a standing and kneeling posture under various pressure of the water pump were measured via the standardized electromyographic activities of altogether 8 muscles of the hand-arm-shoulder system. These results as well as the subjective experiences documented in a standardized questionnaire of the sample of 12 professional fire-fighters who participated in the comprehensive tests, give evidence of physically bearable and safe indoor fire-fighting in multi-story buildings/high-rises and large properties. 
Electromyography and subjective assessments based on work experiences were again used as the most appropriate methods to compare a newly developed and ergonomically promising computer input device to a standard computer mouse. Kluth and Keller carried out a lot of standardized working tests with a sample of 24 subjects to measure the muscular strain in the hand-arm-shoulder system associated with the two input devises and to prove the ergonomic quality of the products. In all assessed criteria, the rollerbar system was consistently rated more favorably than the standard mouse whereas - due to a rather low level of physical strain during the working tests - the electromyographically quantified physiological costs of the muscles could not be used as indices for an unequivocally preferred input device. There exist also some differences along age and gender lines with regard to the strength of preference.

Serafin et al. report about the physical strength of a population sample and show quite interesting differences in age, gender and hand preference, which are important for the design of products and workplaces. Isometric maximum force measurements (one-handed pulling, gripping, exerting pronation and supination torque strength during inward and outward rotation of the arm while e. g. handling screwdrivers) were carried out with more than 1000 males and females in various age groups. The results showed a distinct hand preference when trying to reach the maximum force level. But surprisingly the results do not differ as significantly as expected in the age groups between 20 and 59 years. However, again the data confirm that females exhibit around $2 / 3$ of the strength level of males.

The paper by Gebhardt et al. which like the $4^{\text {th }}$ paper is a contribution of the Institute of Occupational Health, Safety and Ergonomics of the Wuppertal University, Germany, is focused on anthropometric considerations for designing an artificial test finger as an efficient checking tool to avoid electrical and mechanical hazards. The dimensions of the most common test finger, mentioned in several standards, have remained unchanged for many decades, although the corresponding anthropometric dimensions of the human being as well as the safety standard and safety awareness have changed during the past 50 years. According to the results of the investigation, an adjustment of the test finger length to at least 110 $\mathrm{mm}$ seems to be necessary, reflecting the potential pass-through length.

Guest Editors

Prof. Dr. Helmut Strasser

Prof. Dr. Karsten Kluth Ergonomics Division University of Siegen, Germany E-mail: h.strasser@aws.mb.uni-siegen.de 